\title{
RETRACTION
}

\section{Detection of Ocean Wave Parameters Using Synthetic Aperture Radar (SAR) Data - RETRACTION}

\author{
Sudhir Kumar Chaturvedi, Palanisamy Shanmugam, Chan-Su Yang \\ and Ugur Guven
}

doi:10.1017/S0373463312000562, Published by Cambridge University Press, 17 December 2012.

It has come to our attention that the paper 'Detection of Ocean Wave Parameters Using Synthetic Aperture Radar (SAR) Data' published in Volume 66 Issue 2 of The Journal of Navigation was published without the knowledge or permission of two of the listed authors (Palanisamy Shanmugam and Chan-Su Yang), and furthermore that the article bears close resemblance to an article previously published in The Korean Journal of Remote Sensing Volume 27 under the title 'Retrieval of Spherical Ocean Wave Parameters Using RADARSAT-2 SAR Sensor Observed as Chull, Micronesia'. This constitutes a breach of the Royal Institute of Navigation's and Cambridge University Press's publication policy, and the paper has therefore been retracted. The Editor-in-Chief of The Journal of Navigation would like to make it clear that neither Palanisamy Shanmugam nor Chan-Su Yang had any knowledge of or part in this breach of publication policy.

\section{REFERENCE}

Chaturvedi, S.K., Shanmugam, P., Yang, C.S. and Guven, U. (2013). Detection of Ocean Wave Parameters Using Synthetic Aperture Radar (SAR) Data. The Journal of Navigation, 66, 283-293. 